\title{
Intelligent Line Follower Robot using MSP430G2ET for Industrial Applications
}

\author{
${ }^{1}$ Sourav Sutradhar, ${ }^{* 2}$ Viswanatha $\mathbf{V},{ }^{3}$ Siddhant Kumar, ${ }^{4}$ Shivam Kumar \\ ${ }^{1,2,3,4}$ Department of Electronics and Communication Engineering, Acharya Institute of Technology, Bangalore \\ Email:sourav.beec.15@acharya.ac.in,viswanathav@acharya.ac.in, siddhant.beec.15@acharya.ac.in , \\ shivamkumar.beec.14@acharya.ac.in
}

Received: 06th December 2019, Accepted: 20th January 2020, Published: 30th April 2020

\begin{abstract}
In this article, intelligent line follower is designed and implemented using MSP430G2ET controller which is industry standard controller since it has much more capable libraries.

It detects black line which the robot has to follow using infrared sensors on a white surface with a high contrasted color. Unlike normal black line follower, this robot is featured with obstacle detection and stop with alert using ultrasonic sensor and buzzer until its removed on black line which is predefined path for it and hence prevent itself from getting damaged by the obstacle on the predefined path. Unlike this, if any wireless protocol is used to stop upon finding obstacle, efficiency of the system degrades with draining of battery. This is an efficient with usage of ultrasonic sensor and robust with industry standard controller. This type of systems can perform lot of tasks in industries, like material handling and replace traditional conveyer belts. It also finds application in domestic tasks like health care management.
\end{abstract}

\author{
Keywords \\ Autonomous System, Line Follower, Obstacle Detection, MSP430G2ET, Robot
}

\section{Introduction}

For efficient work in industries, proper monitoring is always needed in work environment for better results and it reduces human efforts in industries. This system can be used to carry ant materials from one place to another place. Unlike conventional robots, the intelligent robot is efficient and more useful since it functionality is automatic i.e. once it finds the predefined path, it keeps moving automatically thus it's called fit and forget ability[ 1]. There are various factors are considered to develop the intelligent line follower. The first one is the conventional obstacle avoiding robot cannot help in transportation of goods since no particular path is defined for it [2]. Such robots will not reach the desired place as it moves randomly while avoiding the obstacles. In addition such robots are not controllable. If any wireless protocols are used for controlling it in real time applications, efficiency of the systems reduces and it can go in any directions and it needs manual operation [3].Therefore intelligent line followers needs to be designed to overcome such problems. The robot detects obstacles using ultrasonic sensor. It's the best and easiest way to find the proximity and levels with high reliability. The care to be taken such that at what environments this sensor works and what not as it uses ultrasonic sound waves which are basically high frequency waves produces echo patterns by reflection from boundaries. The frequency of these sound waves is above the range of human hearing. The sensor is built with transmitter which sends the pulse and the receiver which receives the echo reflected [4]-[9]. The intelligent line follower is safe and reliable system since it can be controlled if anything comes in between predefined path and hence this system can be used in carrying the children in shopping malls and homes. There are some calibration issues associated with analog sensors used with track detection and follow so PWM control signal set to $20 \%$ and $80 \%$ to determine how far away the sensor is from the line and hence the analog value would drop to $50 \%$ of its maximum range. There are the line detection algorithms like (a) line detection via quadratic interpolation (b)Line detection via Weighted Average (c) Proportional integral derivative(PID) controller. As the steady state error is small, integral term is not used. The PD controller used as shown in fig.1.It finds the error between the track of our interest and the center of the reflective optical sensors. The right and left wheels receives the commands by the PD functions. The function "P" improves amplifier gain and function " $D$ " improves the response to the disturbance. The term " $D$ " used to stabilize the tracking motion. Out of many other algorithms, weighted average line-detection algorithm is the best in line detection applications as it uses mean square errors[10]-[12]. 


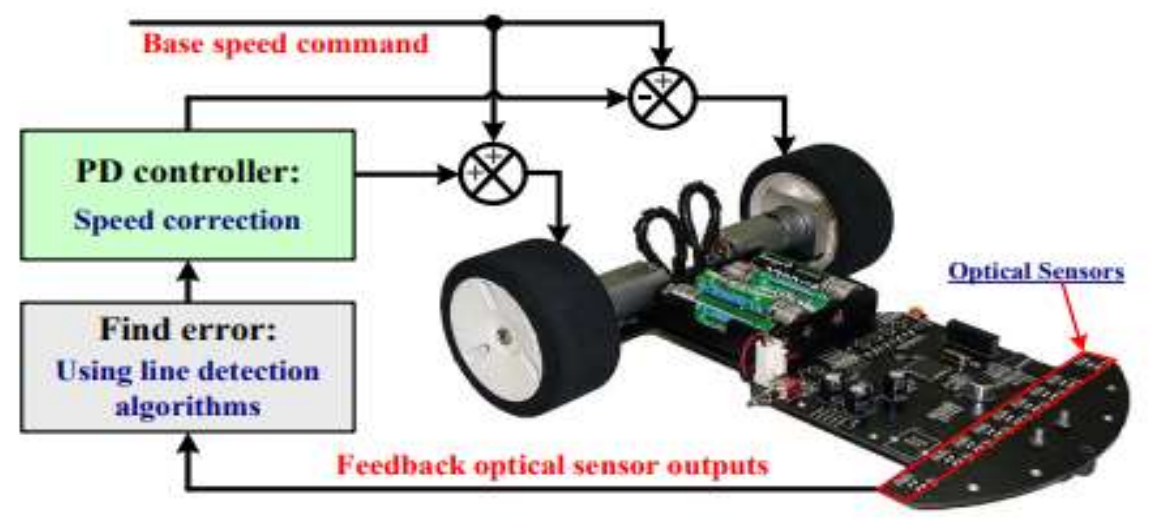

Fig. 1: PD Control Based Intelligent Line following BOT.

\section{Materials \& Methodology \\ IR Sensor}

It's kind of sensor which detects the movement of objects by using the heat generated by them. Its resembles human's visionary senses. It's widely being used in real time applications because of its simple circuitry and easy functionality and interface in electronic systems. This sensor has following four main parts; 1).LM325 IC IR transmitter and receiver pair 2).Resistors of the range of kilo ohms 3).variable resistors 4).Light emitting diode.

IR emitter LED acts as transmitter which keeps on transmitting IR rays. When it falls on the color other than black color, no light gets reflected and hence no signal received by the IR receiver. When the IR rays sent by transmitter falls on black colour,light gets reflected and received by the receiver and hence inverting terminal of the LM325 IC goes low that results in output of the comparator goes high and obstacle LED starts glowing. There are some resistors connected with LM325 comparator circuit to ensure minimum 10mA current passes through obstacle LED. The power LED glows when 5V DC supply is applied between Vcc and GND. When obstacle LED glows, out terminal goes HIGH with 5V.Distance adjust pot is used to set the sensitivity of the circuit.

\section{Ultrasonic Sensor}

It is used to detect the obstacles present on the predefined path. It has four terminals;Vcc.Trigger,Echo and Ground. It works with $+5 \mathrm{~V}$ and $15 \mathrm{~mA}$.measuring angle covers less than $15^{\circ}$, operates with $40 \mathrm{~Hz}$.therritically it measures $2 \mathrm{~cm}$ to $450 \mathrm{~cm}$ but practically it works with $2 \mathrm{~cm}$ to $80 \mathrm{~cm}$. This sensor needs predefined clock signal to emit ultrasonic signal over trigger terminal. When that signal gets objected by any material, it gets reflected back towards the echo terminal. Using basic formulate Distance=speed $\mathrm{x}$ time, the distance is calculated.MSP430 controller measures , how long the echo pulse is high and upon this information it calculate the time taken for the wave to return back to the sensor and calculate the distance.

\section{MSP-EXP430G2ET Launchpad}

The target device in this launch pad is MSP430G2553.It has 24 GPIOs pins .Most of which are multiplexed. This launch pad used as controller in the robot is as shown in fig.2. takes $5 \mathrm{v}$ and through DC-Dc converter present on board will give $3.3 \mathrm{v}$ to the target device. It has one universal serial communication interfaces that supports UART, IrDA and SPI. It also has USCI which supports SPI and $\mathrm{I}^{2} \mathrm{C}$ communication. In hardware implementation of line follower, UART communication is used to program target device. It has eZ-FET debug probe which enables programming and communication back to the computer.it also provides power to the target device by receiving $5 \mathrm{~V}$ from USB and gives $3.3 \mathrm{Vt}$ through $\mathrm{LDO}$.Therfore external programmer is not required to program target device.UART Target device gets frequency of $32.768 \mathrm{KHz}$ from $4 \mathrm{MHz}$ through debug MCU for its speed of operation. In hardware, receiver output signal of left sensor is connected to pin 'p3' and receiver output signal of right sensor is connected to pin 'p4'.Two control signals for two motors are generated with four pins of MSP430.those four pins are $\mathrm{p} 5, \mathrm{p} 6, \mathrm{p} 1$ and $\mathrm{p} 2$. Output of these pins are connected to driver circuit' L298D'. 


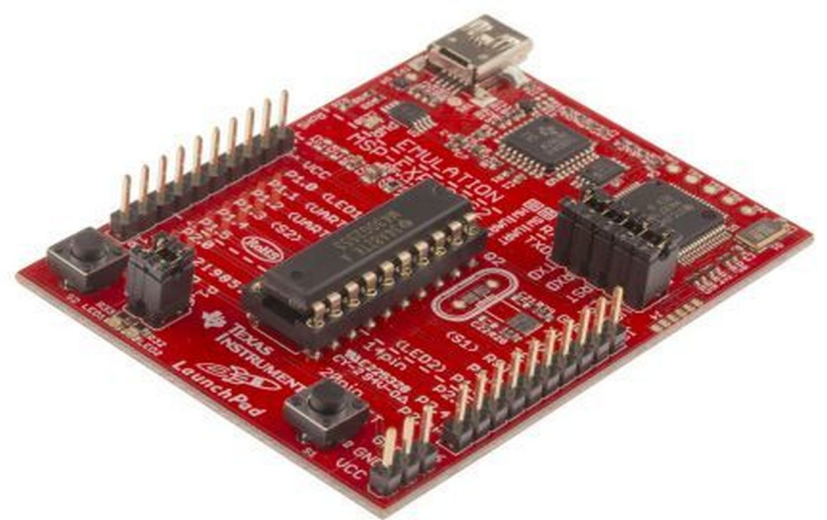

Fig. 2: MSP430G2ET Launchpad

\section{Motor Driver}

The H-bridge motor driver board is used to drive the motor with high voltage. It's basically voltage supplier for the motor based on the logic levels received from the Launchpad. Since motors are rated with high voltage and Launchpad cannot supply such high voltage so this driver supplies required voltage for motors. This particular Board supplies from $5 \mathrm{~V}$ to maximum $45 \mathrm{~V}$.In the hardware implementation $12 \mathrm{~V}$ battery is used to supply motors. It has two outputs; output A for right motor and output B for left motor. Highly Stable DC-DC converters like buck or boost or combinational can be used to drive the motor through the motor driver in order to obtain efficient and stable system since balancing the power for the system of any kind is main concerned issue[13]-[14].

\section{Motors and Wheels}

The geared motor is used as driving elements of the robot. In the implementation, two geared motors are used at the rear side of the robot. These motors provide more torque than conventional motors. These motors capable to carry some loads too.

\section{Methodology}

The black line following robot falls under the category of autonomous robots. The IR sensor used in the bot help to detect and follow the black line .The complete circuit diagram of the BOT is as shown in fig.3. In the implementation, two infrared sensor for the path detection the path may be a black line on a white surface or an white line on a black surface .we have also integrated the bot with an ultrasonic sensor so what happens if any object is detected in the path within the threshold distance the ultrasonic sensor sends the data to the micro controller which trigger the buzzer and the buzzer will keep on buzzing unless and until the object is removed from the path. Hence robot become more intelligent and robust in its functionality.

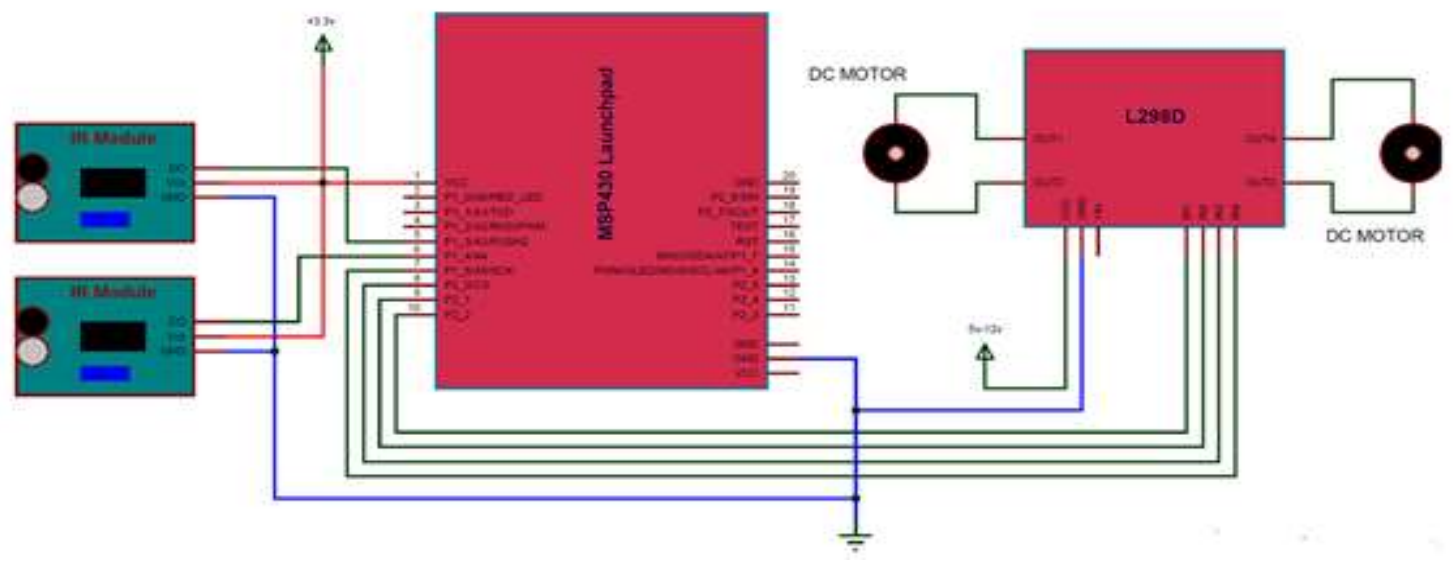

Fig. 3: Complete Circuit Diagram of the Designed Robot 


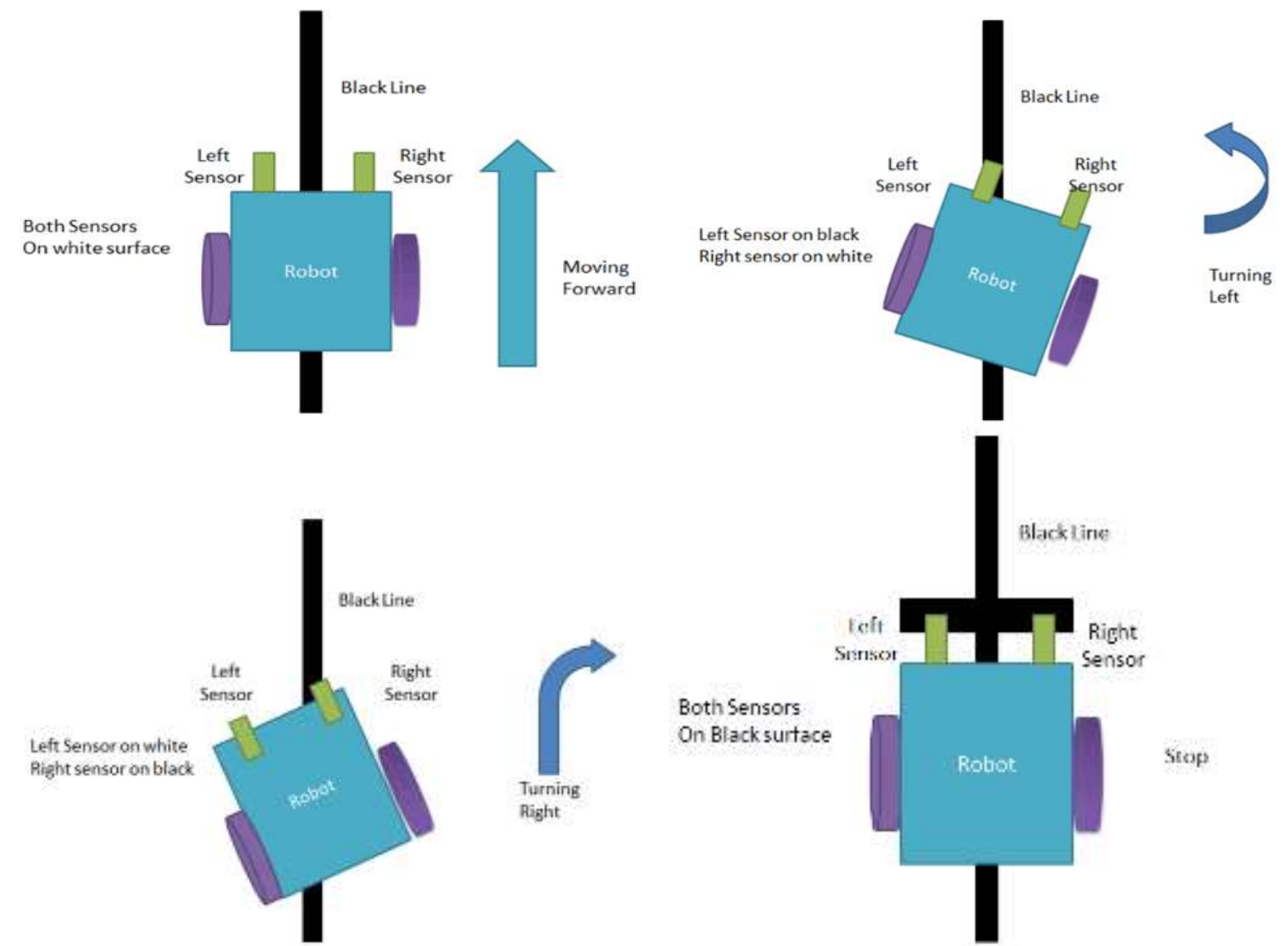

Fig. 4: Control of Robot based on the Logic

When the BOT is positioned on the black line and both the sensor is placed on the white surface the bot moves forward .If the left ir sensor comes over the black line then the bot Moves to its left, if the right infrared sensor falls over the black line then the bot moves to its left .finally when both the sensors are on the black line the bot stops. The control of robot based on the control logic depicted in the table.1 is as shown in the fig.4.

The left sensor and right sensor with GPIO pins P1_3 and P1_4. These two pins act as input pins. The output pins are $\mathrm{P} 1 \_5 \& \mathrm{P} 2 \_0$ which are assigned to the left motor whereas P2_1\&P2_2 are assigned to the right motor. For buzzer interfacing we have allocated the triggerpin, echopin and buzzpin with the MSP430 pin no P2_3,P2_4,P2_5.There are two function used in the program the setup function, followed by the loop function in setup function we have successfully assigned the input and output pin variables. To declare the input/output pins we have used the pinMode function. Left sensor(ls) and right sensor(rs) is taken as input, whereas LM1,LM2,RM1, RM2 are taken as output .This completes our setup function. After the setup function we have the loop function .Inside the loop function we have declared two integer variables, they are duration and distance. Then we have used a formula to calculate the distance and store the value in distance variable. Now we will accord a threshold value and within an if block we will be checking the condition. IF the distance value matches with the threshold value then the buzzer starts buzzing else there is no buzzing sound. In the next if block we have checked if the distance value is less than $50 \mathrm{~cm}$ if it is so then it enter the loop and executes the following statements, where the bot will be in halt state because all the motors are in low sate. Now coming to the else part it enters the if block checks the condition, if the condition is true it enters the loop executes task which is to move forward or turn left or turn right. In order to turn the bot to the left ,the right motors are made high because the right sensors are on the white surface and to turn the bot to the right the left motors are kept high and when finally when both the sensors senses black line then both the motors are kept low which results in stopping of the BOT.

\section{Result \& Discussion}

The hardware implemented with two IR sensors which is used for detecting the track, one Ultrasonic sensor for detecting the obstacles on the track, $12 \mathrm{~V}$ and 7Ah rated battery,MSP430 based controller and driver board. The complete hardware is built and demonstrated as shown in the fig.5 and verified the logic given in the table.1. 
Table 1: Control Logic

\begin{tabular}{|c|c|c|c|c|c|c|c|}
\hline Obstacle on the Track & \multicolumn{2}{|c|}{ Input } & \multicolumn{4}{|c|}{ Output } & \multirow{3}{*}{$\begin{array}{c}\text { Robot } \\
\text { Movement }\end{array}$} \\
\hline \multirow{6}{*}{ Not present } & Left Sensor1 & Right Sensor1 & Left $\mathrm{N}$ & tor1 & Right & otor1 & \\
\hline & LS1 & RS1 & LM1 & LM2 & RM1 & RM2 & \\
\hline & 0 & 0 & 0 & 0 & 0 & 0 & Stop \\
\hline & 0 & 1 & 1 & 0 & 0 & 0 & Turn Right \\
\hline & 1 & 0 & 0 & 0 & 1 & 0 & Turn Left \\
\hline & 1 & 1 & 1 & 0 & 1 & 0 & Forward \\
\hline Present & $\mathrm{x}$ & $\mathrm{x}$ & 0 & 0 & 0 & 0 & $\begin{array}{c}\text { Stop and } \\
\text { alert by } \\
\text { sound }\end{array}$ \\
\hline
\end{tabular}

The designed robot will move on the predefined track as per the signals received from the IR sensors which generates signals based on the logic provided no obstacles present on the predefined track and if it finds any obstacles over the track, it will stop moving irrespective of any state of output of both IR sensors. HC-SR04 ultrasonic sensor detect the obstacle present on the track with in maximum distance of $80 \mathrm{~cm}$ with cone angle of $15^{0}-30^{0}$. Transimtter of the sensor transmits 8 cycles of ultrasonic burst pulses at $40 \mathrm{kHz}$ and echo pin goes high until it receives the reflected signal. Duration of the on time of echo pulse is taken into account to calculate the distance of the object present on the track.
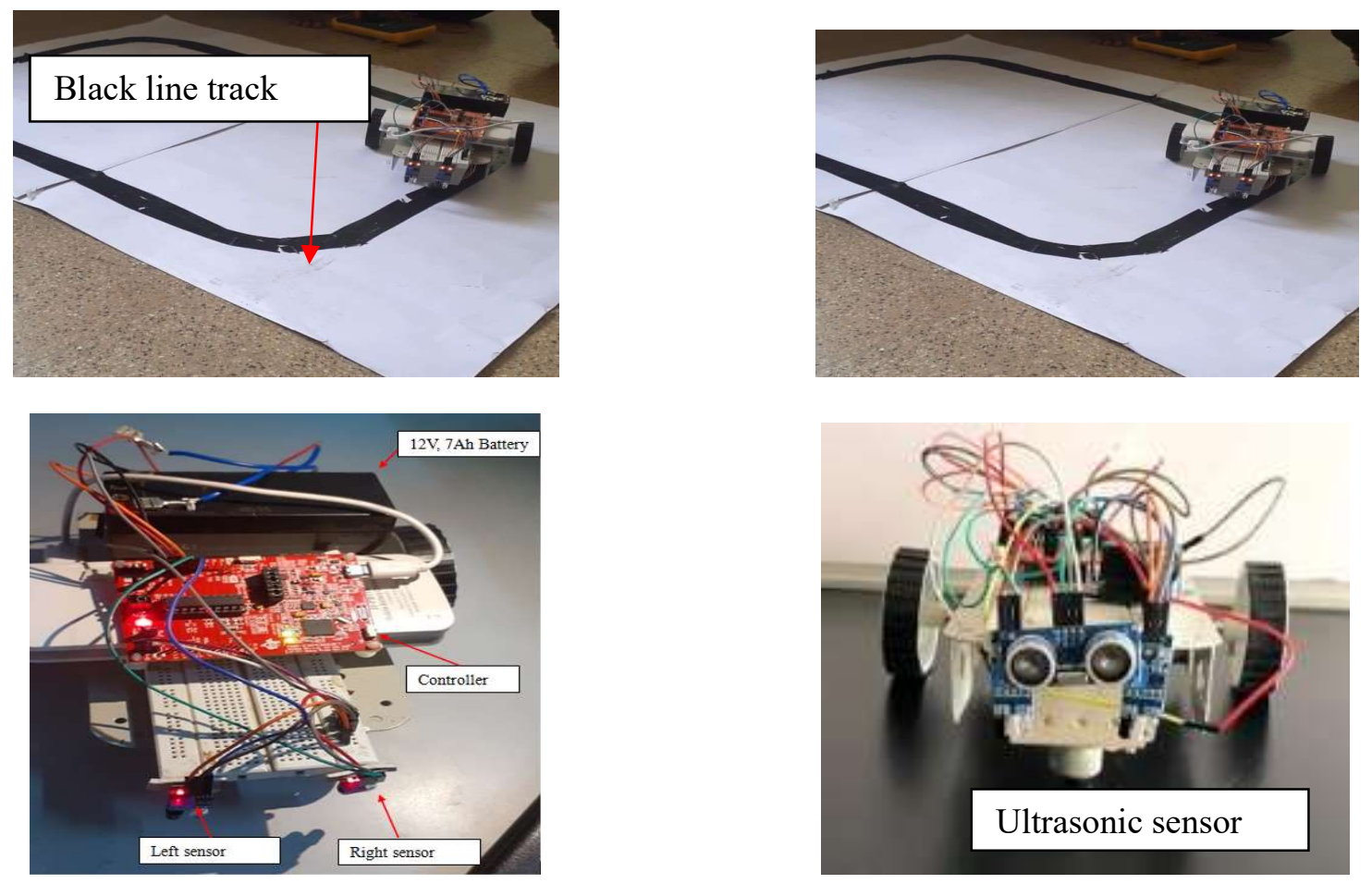

Fig. 5: Hardware Demonstration.

\section{Conclusion}

The main advantages of using MSP430 based families to implement Intelligent BOT over Arduino families are much more capable library in comparison with limited Arduino-clone library ,MSP430 Value line controllers are cheaper than the higher-end ATMega controllers and MSP430 controller can run with the 16Mhz from their internal clock source. The black line follower can be controlled only by changing the path and if any wireless protocol is used to control it, it consumes more power which results in draining of battery quickly. This intelligent BOT has no such problem to control since ultrasonic sensor is used. This system can replace skilled labour with better tasks and accuracy that results in lower per capita cost. 
In future, to take particular path among many paths in the direction of movement of robot, Red, Green, Blue (RGB) color detection sensor can be used in the robot. Robot can differentiate these three different colors and based on the instructions it can choose particular path of our interest that the robot supposed to move.

\section{References}

[1]. Osorio, Román, et al. "Inteligent line follower mini-robot system." International Journal of Computers, Communications \& Control 1.2 (2006): 73-83.

[2]. Punetha, Deepak, Neeraj Kumar, and Vartika Mehta. "Development and applications of line following robot based health care management system." International Journal of Advanced Research in Computer Engineering \& Technology (IJARCET) 2.8 (2013): 2446-2450.

[3]. Ghani, Nor Maniha Abdul, Faradila Naim, and Tan Piow Yon. "Two wheels balancing robot with line following capability." World Academy of Science, Engineering and Technology 55 (2011): 634-638.

[4]. Kumar, Rakesh Chandra, et al. "Obstacle avoiding robot-a promising one." International Journal Of Advances Research In Electrical, Electronics And Instrumentation Engineering 2 (2013).

[5]. Sonal, Gadhvi, Punit Raninga, and Hardik Patel. "Design and implementation of RGB color line following robot." 2017 International Conference on Computing Methodologies and Communication (ICCMC). IEEE, 2017.

[6]. Colak, Ilknur, and Deniz Yildirim. "Evolving a Line Following Robot to use in shopping centers for entertainment." 2009 35th Annual Conference of IEEE Industrial Electronics. IEEE, 2009

[7]. Hussmann, Stephan, and Detlef Jensen. "Crazy car race contest: Multicourse design curricula in embedded system design." IEEE Transactions on Education 50.1 (2007): 61-67.

[8]. Paulik, Mark J., and Mohan Krishnan. "A competition-motivated capstone design course: The result of a fifteen-year evolution." IEEE Transactions on Education 44.1 (2001): 67-75.

[9]. Lee, Chyi-Shyong, et al. "A hands-on laboratory for autonomous mobile robot design courses." IFAC Proceedings Volumes 41.2 (2008): 9743-9748.

[10]. Lee, Chyi-Shyong, et al. "A project-based laboratory for learning embedded system design with industry support." IEEE Transactions on Education 53.2 (2009): 173-181.

[11]. Kumari, Ashwini. "Multilevel Home Security System using Arduino \& GSM." Journal for Research| Volume 4.10 (2018).

[12]. Zimmermann, Hans-Jürgen. Fuzzy set theory—and its applications. Springer Science \& Business Media, 2011.

[13]. Viswanatha, V., and R. Venkata Siva Reddy. "Digital control of buck converter using arduino microcontroller for low power applications." 2017 International Conference on Smart Technologies for Smart Nation (SmartTechCon). IEEE, 2017.

[14]. Viswanatha V, Venkata Siva Reddy R, and Rajeswari. "Stability and Dynamic Response of Analog and Digital Control Loops of Bidirectional Buck-boost Converter for Renewable Energy Applications." International Journal of Recent Technology and Engineering Volume 8.2 (2019): 5181-186. 